Pacific Journal of Mathematics

THE EXACT BERGMAN KERNEL AND THE KERNELS OF
SZEGÓ TYPE 


\title{
THE EXACT BERGMAN KERNEL AND THE KERNELS OF SZEGÖ TYPE
}

\section{SABUROU SAITOH}

\begin{abstract}
A relation between the magnitudes of the exact Bergman kernel and a product of two kernels of Szegö type is given. The method is turned to the establishment of a positive definiteness of a period matrix of a product of two kernels of Szegö type. The positive definiteness leads to some completeness theorems of such products.
\end{abstract}

1. Introduction. Let $G$ denote an $n$-ply connected regular region with boundary components $\left\{C_{\nu}\right\}_{\nu=1}^{n}$. Let $K^{E}\left(z, \bar{z}_{1}\right)$ and $\hat{K}\left(z, \bar{z}_{1}\right)$ denote the exact Bergman kernel and the Szegö kernel of $G$, respectively. Let $\left\{Z_{\nu}(z) d z\right\}_{\nu=1}^{n-1}$ denote a basis of analytic differentials which are real along $\partial G$. Then the following identity is known:

$$
4 \pi \hat{K}\left(z, \bar{z}_{1}\right)^{2}=K^{E}\left(z, \bar{z}_{1}\right)+\sum_{\nu=1}^{n-1} \sum_{\mu=1}^{n-1} C_{\nu \mu} \overline{Z_{\nu}\left(z_{1}\right)} Z_{\mu}(z),
$$

for some uniquely determined constants $C_{\nu \mu}$. D. A. Hejhal [3] established the positive definiteness of the matrix $\left\|C_{\nu \mu}\right\|$ by means of the representations of $C_{\nu \mu}$ in terms of the theta function. In this paper, we shall establish a similar result in a very general situation by means of the pure theory of kernel functions in a sense. Our result leads to a variety of the completeness theorems of the kernels of Szegö type which are established in the paper [6].

In $\S 2$, we state notation and preliminary facts and in $\S 3$, the main theorem is given. In $\S 4$ and $\S 5$, completeness of the products of two kernels of Szegö type is discussed. These two sections are considered as a continuation of the paper [6]. In the final $\S 6$, we refer to the case of the Szegö kernel with characteristic of an arbitrary compact bordered Riemann surface which is established by J. D. Fay [1].

2. Notation and preliminary facts. Let $S$ denote the interior of a compact bordered Riemann surface $\bar{S}$ with boundary contours $\left\{C_{\nu}\right\}_{\nu=2 n+1}^{2 n+m}$ and genus $n$. Let $\left\{C_{\nu}\right\}_{\nu=1}^{2 n+m-1}$ denote a canonical homology basis. Let $M$ denote the Hilbert space of analytic differentials $f(z) d z$ which are regular in $S$ and have finite norms:

$$
\left(\iint_{S}|f(z)|^{2} d x d y\right)^{1 / 2}
$$

$<\infty(z=x+y i)$. Let $K\left(z, \bar{z}_{1}\right) d z$ and $K^{E}\left(z, \bar{z}_{1}\right) d z$ denote the Bergman 
kernel of class $M$ and the exact Bergman kernel of $S$, respectively. Let $L\left(z, z_{1}\right) d z$ and $L^{E}\left(z, z_{1}\right) d z$ denote the adjoint $L$-kernels of theirs, respectively. They are analytic on $\bar{S}$ except for $z_{1}$ where they have a double pole:

$$
\left\{\frac{1}{\pi} \frac{1}{\left(z-z_{1}\right)^{2}}+\text { regular terms }\right\} d z .
$$

Further they satisfy the following relations:

$$
-\overline{K\left(z, \bar{z}_{1}\right) d z}=L\left(z, z_{1}\right) d z \text { and }-\overline{K^{E}\left(z, \bar{z}_{1}\right) d z}=L^{E}\left(z_{1}, z\right) d z \text { along } \partial S .
$$

The $K$-kernels $K\left(z, \bar{z}_{1}\right)$ and $K^{E}\left(z, \bar{z}_{1}\right)$ are Hermitian and $L\left(z, z_{1}\right)$ is symmetric, but $L^{E}\left(z, z_{1}\right)$ is not symmetric, in general (cf. [7], pp. 126-137).

Let $W(z, t)$ denote the meromorphic function which has the Green function $g(z, t)$ of $S$ with pole at $t(\in S)$ as the real part of $W(z, t)$. The differential $\operatorname{id} W(z, t)$ is positive along $\partial S$ and has $N=2 n+m-1$ zeros $\left\{t_{\nu}\right\}$ in $S$. For simplicity, we assume that all the zeros $t_{\nu}$ are simple. In other cases, we can modify the following arguments slightly. Further we shall use the same notation for a point on $\bar{S}$ and a fixed local parameter around there. For an arbitrary integer $q$, let $H_{2}^{q}(S)$ denote the Hilbert space of analytic differentials $f(z)(d z)^{q}$ of order $q$ on $S$ with finite norms:

$$
\left(\frac{1}{2 \pi} \int_{\partial S}\left|f(z)(d z)^{q}\right|^{2}(\mathrm{id} W(z, t))^{1-2 q}\right)^{1 / 2}<\infty,
$$

where $f(z)$ means the Fatou boundary value of $f$ at $z \in \partial S$ in the obvious sense. Let $K_{q, t, \rho}\left(z, \bar{z}_{1}\right)(d z)^{q}$ denote the kernel function of the class $H_{2}^{q}(S)$ which is characterized by the following reproducing property:

$$
\begin{gathered}
f\left(z_{1}\right)=\frac{1}{2 \pi} \int_{\partial S} f(z)(d z)^{q} \overline{K_{q, t, \rho}\left(z, \bar{z}_{1}\right)(d z)^{q}} \rho(z)(\operatorname{id} W(z, t))^{1-2 q} \\
\text { for all } f(z)(d z)^{q} \in H_{2}^{q}(S) .
\end{gathered}
$$

Here $\rho$ is a positive continuous function on $\partial S$. Let $L_{q, t, \rho}\left(z, z_{1}\right)(d z)^{1-q}$ denote the adjoint $L$-kernel of $K_{q, t, \rho}\left(z, \bar{z}_{1}\right)(d z)^{q}$. Then $L_{q, t, \rho}\left(z, z_{1}\right)(d z)^{1-q}$ is a meromorphic differential on $S$ of order $1-q$ with one simple pole at $z_{1}$ with residue 1 (in the obvious sense) and satisfies the relation

$$
\begin{aligned}
& \overline{K_{q, t, \rho}\left(z, \bar{z}_{1}\right)(d z)^{q}} \rho(z)(\operatorname{id} W(z, t))^{1-2 q} \\
& \quad=\frac{1}{i} L_{q, t, \rho}\left(z, z_{1}\right)(d z)^{1-q} \text { along } \partial S .
\end{aligned}
$$


We note that $K_{q, t, \rho}\left(z, \bar{z}_{1}\right)$ and $L_{q, t, \rho}\left(z, z_{1}\right)$ are continuous on $\partial S$.

From (2.2), we have

$$
\begin{aligned}
& \overline{K_{q, t, \rho}\left(z, \bar{z}_{1}\right) K_{1-q, t, \rho^{-1}}\left(z, \bar{z}_{1}\right) d z} \\
& \quad=-L_{q, t, \rho}\left(z, z_{1}\right) L_{1-q, t, \rho^{-1}}\left(z, z_{1}\right) d z \text { along } \partial S .
\end{aligned}
$$

Let $\left\{Z_{\nu}(z) d z\right\}_{v=1}^{N}$ denote a basis of analytic differentials on $\bar{S}$ which are real along $\partial S$ such that $Z_{\nu}(z)=\int_{C_{\nu}} L(\zeta, z) d \zeta$. Then from (2.1) and (2.3), we obtain the identities

$$
K_{q, t, \rho}\left(z, \bar{z}_{1}\right) K_{1-q, t, \rho^{-1}}\left(z, \bar{z}_{1}\right)=\pi K^{E}\left(z, \bar{z}_{1}\right)+\sum_{\nu=1}^{N} \sum_{\mu=1}^{N} C_{\nu \mu} \overline{Z_{\nu}\left(z_{1}\right)} Z_{\mu}(z)
$$

and

$$
L_{q, t, \rho}\left(z, z_{1}\right) L_{1-q, t, \rho^{-1}}\left(z, z_{1}\right)=\pi L^{E}\left(z_{1}, z\right)-\sum_{\nu=1}^{N} \sum_{\mu=1}^{N} \overline{G_{\nu \mu}} Z_{\nu}\left(z_{1}\right) Z_{\mu}(z),
$$

for some uniquely determined constants $\left\{C_{\nu \mu}\right\}$. Our first objective is to show the positive definiteness of the matrix $\left\|C_{\nu \mu}\right\|$. By setting $\int_{C_{\mu}} Z_{\nu}(z) d z=P_{\nu \mu}$, from (2.4), we obtain

$$
\begin{gathered}
\sum_{\nu} \sum_{\mu} C_{\nu \mu} \overline{P_{\nu \alpha}} P_{\mu \beta}=\int_{C_{\alpha}}\left(\int_{C_{\beta}} K_{q, t, \rho}\left(z, \bar{z}_{1}\right) K_{1-q, t, \rho^{-1}}\left(z, \bar{z}_{1}\right) d z\right) \overline{d z_{1}} \\
\text { for } \alpha, \beta=1,2,3, \cdots, N .
\end{gathered}
$$

Since the matrix $\left\|P_{\nu \mu}\right\|$ is nonsingular (cf. [7], pp. 93-97 and pp. 109-110), we shall show the positive definiteness of (2.6).

Here we note that especially $K_{0, t, 1}\left(z, \bar{z}_{1}\right)$ and $K_{1, t, 1}\left(z, \bar{z}_{1}\right) d z$ are the Rudin kernels (cf. [5]). If $S$ is a bounded regular region on the plane, then we can identify functions and differentials on $S$. Hence we can write the reproducing property of $K_{q, t, \rho}\left(z, \bar{z}_{1}\right)(d z)^{q}$ as follows:

$$
\begin{gathered}
f\left(z_{1}\right)=\frac{1}{2 \pi} \int_{\partial S} f(z) \overline{K_{q, t, \rho}\left(z, \bar{z}_{1}\right)} \rho(z)\left(\frac{\partial g(z, t)}{\partial \nu}\right)^{1-2 q} d s_{z} \\
\text { for all } f \in H_{2}^{0}(S),
\end{gathered}
$$

where $\partial / \partial \nu$ denotes the inner normal derivative with respect to $S$. Therefore we can regard $K_{q, t, \rho}\left(z, \bar{z}_{1}\right)$ as the Szegö kernel with weight $\rho(z)(\partial g(z, t) / \partial \nu)^{1-2 q}$ for the (Hardy) space $H_{2}^{0}(S)$. By this interpretation of (2.7), we can consider the kernel $K_{q, t, \rho}\left(z, \bar{z}_{1}\right)$ for an arbitrary real value of $q$ and $K_{1 / 2, t, 1}\left(z, \bar{z}_{1}\right) / 2 \pi$ is the classical Szegö kernel. For a real value of $q$, we can take a more general interpretation for (2.7) and we shall refer to this in $\S 6$, again. 
3. Positive definiteness of $K_{q, t, \rho}\left(z, \bar{z}_{1}\right) K_{1-q, t, \rho^{-1}}\left(z, \bar{z}_{1}\right)$.

THEOREM 3.1. The matrix

$$
\left\|\int_{C_{\nu}}\left(\int_{C_{\mu}} K_{q, t, \rho}\left(z, \bar{z}_{1}\right) K_{1-q, t, \rho-1}\left(z, \bar{z}_{1}\right) d z\right) \overline{d z_{1}}\right\|^{N \times N}
$$

is positive definite.

Proof. Let $\left\{\phi_{j}\right\}_{j=0}^{\infty}$ and $\left\{\psi_{k}\right\}_{k=0}^{\infty}$ denote some complete orthonormal systems such that $K_{q, t, \rho}\left(z, \bar{z}_{1}\right)=\sum_{j} \overline{\varphi_{j}\left(z_{1}\right)} \varphi_{j}(z)$ and $K_{1-q, t, \rho^{-1}}\left(z, \bar{z}_{1}\right)=$ $\sum_{k} \overline{\psi_{k}\left(z_{1}\right)} \psi_{k}(z)$. Then we have

$$
\begin{array}{r}
\int_{C_{\nu}}\left(\int_{C_{\mu}} K_{q, t, \rho}\left(z, \bar{z}_{1}\right) K_{1-q, t, \rho^{-1}}\left(z, \bar{z}_{1}\right) d z\right) \overline{d z_{1}} \\
=\sum_{j} \sum_{k} \int_{C_{\mu}} \varphi_{j}(z) \psi_{k}(z) d z \overline{\int_{C_{\nu}} \varphi_{j}\left(z_{1}\right) \psi_{k}\left(z_{1}\right) d z_{1}} .
\end{array}
$$

Here we see easily that the double sequence converges absolutely. Let $m$ denote the double index $(j k)$ and we set

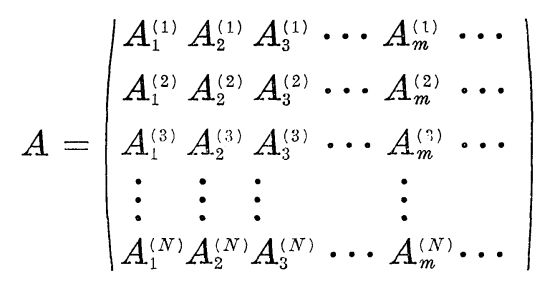

where

$$
A_{m}^{(\nu)}=\int_{C_{\nu}} \varphi_{j}(z) \psi_{k}(z) d z(\nu=1,2,3, \cdots, N) .
$$

Further we set $X=\left(X_{1}, X_{2}, X_{3}, \cdots, X_{N}\right) \in C^{N}$. Then we obtain

$$
X A \bar{A}^{t} \bar{X}^{t}=\sum_{m}\left|X_{1} A_{m}^{(1)}+X_{2} A_{m}^{(2)}+X_{3} A_{m}^{(3)}+\cdots+X_{N} A_{m}^{(N)}\right|^{2} \geqq 0 .
$$

Here equality holds if and only if

$$
X_{1} A_{m}^{(1)}+X_{2} A_{m}^{(2)}+\cdots+X_{N} A_{m}^{(N)}=0 \text { for all } m .
$$

Hence we obtain, for any $z_{1}$ and $z_{2} \in S$,

$$
\sum_{\nu=1}^{N} X_{\nu} \int_{C_{\nu}} K_{q, t, \rho}\left(z, \bar{z}_{1}\right) K_{1-q, t, \rho^{-1}}\left(z, \bar{z}_{2}\right) d z=0 \text {. }
$$

From the identity

$$
K_{q, t, \rho}\left(z, \bar{z}_{1}\right)=\frac{1}{2 \pi} \int_{\partial S} K_{q, t, \rho}\left(\zeta, \bar{z}_{1}\right)(d \zeta)^{q} \overline{K_{q, t, \rho}(\zeta, \bar{z})(d \zeta)^{q}} \rho(\zeta)(\operatorname{id} W(\zeta, t))^{1-2 q},
$$


we have, by exchanging the variables,

$$
\begin{aligned}
& \int_{\partial S}\left[\left(\sum_{2 n+1}^{N} \omega_{\nu}(z) X_{\nu}\right) K_{1-q, t, \rho-1}\left(z, \bar{z}_{2}\right)(d z)^{1-q}\right. \\
& \quad+\left(\frac{1}{2 \pi} \sum_{\nu=1}^{2 n} X_{\nu} \int_{C_{\nu}} \overline{K_{q, t, \rho}(z, \bar{\zeta})(d z)^{q}} K_{1-q, t, \rho-1}\left(\zeta, \bar{z}_{2}\right) d \zeta\right) \\
& \left.\quad \times \rho(z)(\operatorname{id} W(z, t))^{1-2 q}\right] K_{q, t, \rho}\left(z, \bar{z}_{1}\right)(d z)^{q}=0 \text { for all } z_{1} \text { and } z_{2} \in S .
\end{aligned}
$$

Here $\omega_{\nu}(z)$ denotes the harmonic measure of $C_{\nu}$ on $S$. Let $\Omega(z) d z$ be a nonvanishing analytic differential on $\bar{S}$. Then since the set of kernels $\left\{K_{q, t, \rho}\left(z, \bar{z}_{1}\right)(d z)^{q} \mid z_{1} \in S\right\}$ is complete in $H_{2}^{q}(S)$, we have

$$
\begin{aligned}
& \int_{\partial S} f(z) d z {\left[\left(\sum_{2 n+1}^{N} \omega_{\nu}(z) X_{\nu}\right) K_{1-q, t, \rho^{-1}}\left(z, \bar{z}_{2}\right)(d z)^{1-q}\right.} \\
&+\left(\frac{1}{2 \pi} \sum_{\nu=1}^{2 n} X_{\nu} \int_{C_{\nu}} \overline{K_{q, t, \rho}(z, \bar{\zeta})(d z)^{q}} K_{1-q, t, \rho^{-1}}\left(\zeta, \bar{z}_{2}\right) d \zeta\right) \\
&\left.\quad \times \rho(z)(\operatorname{id} W(z, t))^{1-2 q}\right](\Omega(z) d z)^{q-1}=0 \text { for all } f(z) d z \in H_{2}^{1}(S) .
\end{aligned}
$$

Hence from the theorem of Cauchy-Read [4], we obtain, by a function $F_{z_{2}} \in H_{2}^{0}(S)$,

$$
\begin{aligned}
& {\left[\left(\sum_{2 n+1}^{N} \omega_{\nu}(z) X_{\nu}\right) K_{1-q, t, \rho^{-1}}\left(z, \bar{z}_{2}\right)(d z)^{1-q}+\left(\frac{1}{2 \pi} \sum_{\nu=1}^{2 n} X_{\nu} \int_{C_{\nu}} \overline{K_{q, t, \rho}(z, \bar{\zeta})(d z)^{q}}\right.\right.} \\
& \left.\left.\quad \times K_{1-q, t, \rho^{-1}}\left(\zeta, \bar{z}_{2}\right) d \zeta\right) \rho(z)(\operatorname{id} W(z, t))^{1-2 q}\right](\Omega(z) d z)^{q-1}=F_{z_{2}}(z) \text { a.e. on } \partial S .
\end{aligned}
$$

From the relation (2.2),

$$
\begin{aligned}
& {\left[\left(\sum_{2 n+1}^{N} \omega_{\nu}(z) X_{\nu}\right) K_{1-q, t, \rho^{-1}}\left(z, \bar{z}_{2}\right)(d z)^{1-q}+\frac{1}{2 \pi i} \sum_{\nu=1}^{2 n} X_{\nu} \int_{C_{\nu}} L_{q, t, \rho}(z, \zeta)(d z)^{1-q}\right.} \\
& \left.\quad \times K_{1-q, t, \rho^{-1}}\left(\zeta, \bar{z}_{2}\right) d \zeta\right](\Omega(z) d z)^{q-1}=F_{z_{2}}(z) \text { a.e. on } \partial S
\end{aligned}
$$

At first, from the Lusin-Riesz-Privalow theorem, we see that all the $\left\{X_{\nu}\right\}_{\nu=2 n+1}^{N}$ are zero. Next since $L_{q, t, \rho}(z, \zeta)$ has a simple pole at $z=\zeta$, from the property of Cauchy integral, we see that the constants $\left\{X_{\nu}\right\}_{\nu=1}^{2 n}$ are also zero. Thus we have completed the proof of the theorem.

From the theorem, we obtain

CoROLlaRy 3.1. The matrix $\left\|C_{\nu \mu}\right\|$ is positive definite. Especially we have the inequality

$$
K_{q, t, \rho}(z, \bar{z}) K_{1-q, t, \rho^{-1}}(z, \bar{z})>\pi K^{E}(z, \bar{z}) \quad \text { for all } z \in S \text {. }
$$


COROLlaRY 3.2. The periods as anti-analytic differentials in $z_{1} \in S$

$$
\left\{\int_{C_{\nu}} K_{q, t, \rho}\left(z, \bar{z}_{1}\right) K_{1-q, t, \rho-1}\left(z, \bar{z}_{1}\right) d z\right\}_{\nu=1}^{N}
$$

are linearly independent.

Here we note that Theorem 3.1 and its corollaries are valid for an arbitrary real value of $q$, when $S$ is a bounded regular region in the plane and we regard $K_{q, t, \rho}\left(z, \bar{z}_{1}\right)$ as the weighted Szegö kernel.

4. Completeness of $\left\{K_{q, t, \rho}\left(z, \bar{z}_{1}\right) K_{1-q, t, \rho^{-1}}\left(z, \bar{z}_{1}\right) d z \mid z_{1} \in S\right\}$ in $M$. Let $\left\{Z_{j}\right\}_{j=1}^{\infty}\left(Z_{j} \neq Z_{j^{\prime}}\right.$, for $\left.j \neq j^{\prime}\right)$ denote any point set of $S$ such that $\lim _{j \rightarrow \infty} Z_{j}=Z_{0}$ for some $Z_{0} \in S$. Then, as we see from the reproducing property, the set of kernels $\left\{K_{q, t, \rho}\left(z, \bar{Z}_{j}\right)(d z)^{q}\right\}_{j=1}^{\infty}$ is complete in $H_{2}^{q}(S)$. In the following two sections we shall show that the set $\left\{K_{q, t, \rho}\left(z, \bar{Z}_{j}\right) K_{1-q, t, \rho^{-1}}\left(z, \bar{Z}_{j}\right) d z\right\}_{j=1}^{\infty}$ is complete in both $M$ and $H_{2}^{1}(S)$. These theorems are a variety of the completeness theorems which are given in the paper [6].

As in the representations (2.4) and (2.5), we obtain

$$
K_{q, t, \rho}\left(z, \bar{z}_{1}\right) K_{1-q, t, \rho-1}\left(z, \bar{z}_{1}\right)=\pi K\left(z, \bar{z}_{1}\right)+\sum_{\nu=1}^{N} \sum_{\mu=1}^{N} C_{\nu}^{*} \overline{Z_{\nu}\left(z_{1}\right)} Z_{\mu}(z)
$$

and

$$
L_{q, t, \rho}\left(z, z_{1}\right) L_{1-q, t, \rho-1}\left(z, z_{1}\right)=\pi L\left(z, z_{1}\right)-\sum_{\nu=1}^{N} \sum_{\mu=1}^{N} C_{\nu \mu}^{*} Z_{\nu}\left(z_{1}\right) Z_{\mu}(z) .
$$

Here we note that the constants $C_{\nu \mu}^{*}$ are real symmetric by virtue of Hermitian of the $K$-kernels and symmetry of $L_{q, t, \rho}\left(z, z_{1}\right) L_{1-q, t, \rho^{-1}}\left(z, z_{1}\right)$ and $L\left(z, z_{1}\right)$. Note that $L_{q, t, \rho}\left(z, z_{1}\right)=-L_{1-q, t, \rho^{-1}}\left(z_{1}, z\right)([5])$. As to the constants $C_{\nu \mu}^{*}$, we obtain

Lemma 4.1. The matrix

$$
\left\|\sum_{\alpha} C_{\nu \alpha}^{*} P_{\alpha \mu}-\pi \delta_{\nu \mu}\right\|^{N \times N}
$$

is nonsingular.

Proof. Suppose that

$$
\begin{aligned}
& \sum_{\mu} Y_{\mu} \int_{C_{\mu}} K_{q, t, \rho}\left(z, \bar{z}_{1}\right) K_{1-q, t, \rho^{-1}}\left(z, \bar{z}_{1}\right) d z \\
& \quad=\sum_{\mu} Y_{\mu} \int_{C_{\mu}}\left[\pi K\left(z, \bar{z}_{1}\right)+\sum_{\alpha} \sum_{\beta} C_{\alpha \beta}^{*} \overline{Z_{\alpha}\left(z_{1}\right)} Z_{\beta}(z)\right] d z \equiv 0 .
\end{aligned}
$$


Then from the identity

$$
\begin{aligned}
\int_{C_{\mu}} K\left(z, \bar{z}_{1}\right) d z & =-\iint_{S} K\left(z, \bar{z}_{1}\right) \overline{Z_{\mu}(z)} d x d y \\
& =-\overline{Z_{\mu}\left(z_{1}\right)} \text { (cf. [7], pp. 102-105), }
\end{aligned}
$$

we have

$$
-\sum_{\mu} \pi Y_{\mu} \overline{Z_{\mu}\left(z_{1}\right)}+\sum_{\mu} \sum_{\alpha} \sum_{\beta} Y_{\mu} C_{\alpha \beta}^{*} \overline{Z_{\alpha}\left(z_{1}\right)} P_{\beta \mu} \equiv 0
$$

Hence we obtain

$$
-\pi Y_{\alpha}+\sum_{\beta} \sum_{\mu} C_{\alpha \beta}^{*} P_{\beta \mu} Y_{\mu}=0 \quad \text { for } \quad \alpha=1,2,3, \cdots, N .
$$

Thus Corollary 3.2 is equivalent to the lemma.

Now we obtain

THEOREM 4.1. The set $\left\{K_{q, t, \rho}\left(z, \bar{Z}_{j}\right) K_{1-q, t, \rho^{-1}}\left(z, \bar{Z}_{j}\right) d z\right\}_{j}$ is complete in $M$.

Proof. Suppose that for any $f(z) d z \in M$,

$$
\iint_{S} f(z) \overline{K_{q, t, \rho}\left(z, \bar{Z}_{j}\right) K_{1-q, t, \rho-1}\left(z, \bar{Z}_{j}\right)} d x d y=0 \quad \text { for all } j \text {. }
$$

Hence from (4.1) and the identity

$$
\iint_{S} f(z) \overline{Z_{\mu}(z)} d x d y=-\int_{C_{\mu}} f(z) d z \text { (cf. [7], p. 102), }
$$

we have

$$
\pi f\left(z_{1}\right) \equiv \sum_{\alpha} \sum_{\beta} C_{\alpha \beta}^{*} Z_{\alpha}\left(z_{1}\right) \int_{C_{\beta}} f(z) d z
$$

Thus from the identities

$$
\pi \int_{C_{\mu}} f\left(z_{1}\right) d z_{1}=\sum_{\alpha} \sum_{\beta} C_{\alpha \beta}^{*} P_{\alpha \mu} \int_{C_{\beta}} f(z) d z \mu=1,2,3, \cdots, N,
$$

and Lemma 4.1, we obtain the desired result.

5. Completeness of $\left\{K_{q, t, \rho}\left(z, \bar{Z}_{j}\right) K_{1-q, t, \rho^{-1}}\left(z, \bar{Z}_{j}\right) d z\right\}_{j}$ in $H_{2}^{1}(S)$. The following lemma is essential for our purpose:

LeMma 5.1. For the critical points $\left\{t_{\nu}\right\}_{\nu=1}^{N}$ of the Green function $g(z, t)$, the matrix

$$
\left\|\int_{C_{\mu}} L_{q, t, \rho}\left(z, t_{\nu}\right) L_{1-q, t, \rho-1}\left(z, t_{\nu}\right) d z\right\|^{N \times N}
$$


is nonsingular.

If not all $t_{\nu}$ is simple, we modify the above matrix slightly, as usual.

Proof. We proceed as in Theorem 2.1 in [6], but in this case the proof is more delicate. From the reproducing property of $K_{1, t, 1}\left(z, \bar{z}_{1}\right) d z$, we have

$$
\begin{aligned}
& K_{q, t, \rho}\left(z_{1}, \bar{z}\right) K_{1-q, t, \rho^{-1}}\left(z_{1}, \bar{z}\right) \\
& \quad=\frac{1}{2 \pi} \int_{\partial S} \frac{K_{q, t, \rho}(\zeta, \bar{z}) K_{1-q, t, \rho^{-1}}(\zeta, \bar{z}) d \zeta \overline{K_{1, t, 1}\left(\zeta, \bar{z}_{1}\right) d \zeta}}{\operatorname{id} W(\zeta, t)} .
\end{aligned}
$$

Hence from (2.3) and the residue theorem, we obtain

$$
\begin{aligned}
& \left(\frac{K_{1, t, 1}\left(z, \bar{z}_{1}\right)}{W^{\prime}(z, t)}\right)^{\prime}=-K_{q, t, \rho}\left(z, \bar{z}_{1}\right) K_{1-q, t, \rho-1}\left(z, \bar{z}_{1}\right) \\
& \quad-\sum_{\nu}\left(\frac{K_{1, t, 1}\left(t_{\nu}, \bar{z}_{1}\right)}{W^{\prime \prime}\left(t_{\nu}, t\right)}\right) L_{q, t, \rho}\left(z, t_{\nu}\right) L_{1-q, t, \rho^{-1}}\left(z, t_{\nu}\right),
\end{aligned}
$$

and

$$
\begin{aligned}
& K_{1, t, 1}\left(z, \bar{z}_{1}\right)=-\left\{\int_{t}^{z} K_{q, t, \rho}\left(\zeta, \bar{z}_{1}\right) K_{1-q, t, \rho^{-1}}\left(\zeta, \bar{z}_{1}\right) d \zeta\right. \\
& \left.\quad+\sum_{\nu} \frac{K_{1, t, 1}\left(t_{\nu}, \bar{z}_{1}\right)}{W^{\prime \prime}\left(t_{\nu}, t\right)} \int_{t}^{z} L_{q, t, \rho}\left(\zeta, t_{\nu}\right) L_{1-q, t, \rho^{-1}}\left(\zeta, t_{\nu}\right) d \zeta\right\} W^{\prime}(z, t)
\end{aligned}
$$

From (5.1), we obtain

$$
\begin{aligned}
& \sum_{\nu} \frac{K_{1, t, 1}\left(t_{\nu}, \bar{z}_{1}\right)}{W^{\prime \prime}\left(t_{\nu}, t\right)} \int_{C_{\mu}} L_{q, t, \rho}\left(z, t_{\nu}\right) L_{1-q, t, \rho^{-1}}\left(z, t_{\nu}\right) d z \\
& \quad=-\int_{C_{\mu}} K_{q, t, \rho}\left(z, \bar{z}_{1}\right) K_{1-q, t, \rho^{-1}}\left(z, \bar{z}_{1}\right) d z \mu=1,2,3, \cdots, N .
\end{aligned}
$$

Let $\left\{X_{\nu}\right\}$ be any solution of system (5.3); i.e.,

$$
\begin{aligned}
& \sum_{\nu} X_{\nu} \int_{C_{\mu}} L_{q, t, \rho}\left(z, t_{\nu}\right) L_{1-q, t, \rho-1}\left(z, t_{\nu}\right) d z \\
& \quad=-\int_{C_{\mu}} K_{q, t, \rho}\left(z, \bar{z}_{1}\right) K_{1-q, t, \rho-1}\left(z, \bar{z}_{1}\right) d z \mu=1,2,3, \cdots, N,
\end{aligned}
$$

and we shall define

$$
\begin{aligned}
\widetilde{K}_{1, t, 1}\left(z, \bar{z}_{1}\right) & =-\left\{\int_{t}^{z} K_{q, t, \rho}\left(\zeta, \bar{z}_{1}\right) K_{1-q, t, \rho^{-1}}\left(\zeta, \bar{z}_{1}\right) d \zeta\right. \\
& \left.+\sum_{\nu} X_{\nu} \int_{t}^{z} L_{q, t, \rho}\left(\zeta, t_{\nu}\right) L_{1-q, t, \rho^{-1}}\left(\zeta, t_{\nu}\right) d \zeta\right\} W^{\prime}(z, t) .
\end{aligned}
$$

Then $\widetilde{K}_{1, t, 1}\left(z, \bar{z}_{1}\right) d z \in H_{2}^{1}(S)$ and from the definition of $X_{\nu}$ and the 
identity (2.5), we see that

$$
\left(K_{q, t, \rho}\left(\zeta, \bar{z}_{1}\right) K_{1-q, t, \rho^{-1}}\left(\zeta, \bar{z}_{1}\right)+\sum_{\nu} X_{\nu} L_{q, t, \rho}\left(\zeta, t_{\nu}\right) L_{1-q, t, \rho^{-1}}\left(\zeta, t_{\nu}\right)\right) d \zeta
$$

is exact. (We assume that $t_{\nu} \notin C_{\mu}$ for all $\nu$ and $\mu_{\text {.) }}$ For any analytic differential $f(z) d z$ on $\bar{S}$ (in fact in $S$ such that $f(z) d z \in H_{2}^{1}(S)$ ), we set

$$
\begin{aligned}
& I=\frac{1}{2 \pi} \int_{\partial S} \frac{f(z) d z \overline{\widetilde{K}_{1, t, 1}\left(z, \bar{z}_{1}\right) d z}}{\operatorname{id} W(z, t)} \\
& =\frac{1}{2 \pi i} \int_{\partial S} f(z)\left[\overline{\int_{t}^{2}\left(K_{q, t, \rho}\left(\zeta, \bar{z}_{1}\right) K_{1-q, t, \rho^{-1}}\left(\zeta, \bar{z}_{1}\right)\right.}\right. \\
& \left.\overline{\left.+\sum_{\nu} X_{\nu} L_{q, t, \rho}\left(\zeta, t_{\nu}\right) L_{1-q, t, \rho^{-1}}\left(\zeta, t_{\nu}\right)\right) d \zeta}\right] d z \text {. }
\end{aligned}
$$

Let $D_{\nu}$ be a tiny disc of radius $r_{\nu}$ in the plane of a local parameter at $t_{\nu}$, with center $t_{\nu}$ and let $D$ denote the union of the $D_{\nu}$, $\nu=1,2,3, \cdots, N$. Then from the Green's formula, we obtain

$$
\begin{aligned}
& I-\sum_{\nu} \frac{1}{2 \pi i} \int_{\partial D_{\nu}} f(z)\left[\overline{\left[\int _ { t } ^ { z } \left(K_{q, t, \rho}\left(\zeta, \bar{z}_{1}\right) K_{1-q, t, \rho^{-1}}\left(\zeta, \bar{z}_{1}\right)\right.\right.}\right. \\
& \left.\overline{\left.+\sum_{\nu} X_{\nu} L_{q, t, \rho}\left(\zeta, t_{\nu}\right) L_{1-q, t, \rho^{-1}}\left(\zeta, t_{\nu}\right)\right) d \zeta}\right] d z \\
& =\frac{1}{\pi} \iint_{S-D} f(z) \overline{\left(K_{q, t, \rho}\left(z, \bar{z}_{1}\right) K_{1-q, t, \rho^{-1}}\left(z, \bar{z}_{1}\right)\right.} \\
& \overline{\left.+\sum_{\nu} X_{\nu} L_{q, t, \rho}\left(z, t_{\nu}\right) L_{1-q, t, \rho^{-1}}\left(\boldsymbol{z}, t_{\nu}\right)\right)} d x d y \text {. }
\end{aligned}
$$

By letting $\sum_{\nu}\left|r_{\nu}\right|$ tend to zero, we obtain

$$
\begin{aligned}
I= & \frac{1}{\pi} \iint_{S} f(z) \overline{K_{q, t, \rho}\left(z, \bar{z}_{1}\right) K_{1-q, t, \rho^{-1}}\left(z, \bar{z}_{1}\right)} d x d y \\
& +\frac{1}{\pi} \sum_{\nu} \overline{X_{\nu}} p_{t_{\nu}} v \iiint_{S} f(z) \overline{L_{q, t, \rho}\left(z, t_{\nu}\right) L_{1-q, t, \rho^{-1}}\left(z, t_{\nu}\right)} d x d y,
\end{aligned}
$$

(cf. (2.5) and [7], pp. 118-120). Hence if we can show that $I=f\left(z_{1}\right)$, we obtain the lemma as in Theorem 2.1 in [6].

From (4.1), (4.2), (4.3), and the fact

$$
\begin{aligned}
& \text { p.v. } \iint_{S} f(z) \overline{L(z, \xi)} d x d y=0 \\
& \quad \text { for all } f(z) d z \in M \text { (cf. [7], pp. 121-126), }
\end{aligned}
$$

we have 


$$
\begin{aligned}
I=f\left(z_{1}\right) & +\frac{1}{\pi}\left[\sum_{\alpha} \sum_{\beta} C_{\alpha \beta}^{*} Z_{\alpha}\left(z_{1}\right)\left(-\int_{C_{\beta}} f(z) d z\right)\right. \\
& \left.-\sum_{\nu} \sum_{\alpha} \sum_{\beta} \overline{X_{\nu}} C_{\alpha \beta}^{*} \overline{Z_{\alpha}\left(t_{\nu}\right)}\left(-\int_{C_{\beta}} f(z) d z\right)\right] .
\end{aligned}
$$

Hence we must show that

$$
\sum_{\alpha} C_{\alpha \beta}^{*} Z_{\alpha}\left(z_{1}\right)-\sum_{\alpha} \sum_{\nu} \overline{X_{\nu}} C_{\beta \alpha}^{*} \overline{Z_{\alpha}\left(t_{\nu}\right)}=0 \quad \beta=1,2,3, \cdots, N .
$$

From the definition of $X_{\nu}$, by making use of (4.1) and (4.2), we obtain

$$
\begin{gathered}
\pi \sum_{\nu} X_{\nu} Z_{\mu}\left(t_{\nu}\right)-\sum_{\nu} \sum_{\alpha} \sum_{\beta} X_{\nu} C_{\alpha \beta}^{*} Z_{\alpha}\left(t_{\nu}\right) P_{\beta \mu} \\
=\pi \overline{Z_{\mu}\left(z_{1}\right)}-\sum_{\alpha} \sum_{\beta} C_{\alpha \beta}^{*} \overline{Z_{\alpha}\left(z_{1}\right)} P_{\beta \mu}
\end{gathered}
$$

and hence

$$
\begin{aligned}
& \pi\left(\sum_{\nu} X_{\nu} Z_{\mu}\left(t_{\nu}\right)-\overline{Z_{\mu}\left(z_{1}\right)}\right) \\
& \quad-\sum_{\beta} \sum_{\alpha}\left(\sum_{\nu} X_{\nu} Z_{\alpha}\left(t_{\nu}\right)-\overline{\left.Z_{\alpha}\left(z_{1}\right)\right)} C_{\alpha \beta}^{*} P_{\beta \mu}=0 \quad \mu=1,2,3, \cdots, N .\right.
\end{aligned}
$$

Hence from Lemma 4.1, we obtain

$$
\sum_{\nu} X_{\nu} Z_{\mu}\left(t_{\nu}\right)-\overline{Z_{\mu}\left(z_{1}\right)}=0 \quad \mu=1,2,3, \cdots, N
$$

Thus from the regularity of the matrix $\left\|P_{\beta \mu}\right\|$ and (5.5), we obtain the desired result (5.4).

Now as in the proof of Theorem 2.2 in [6], we obtain

THEOREM 5.1. The set $\left\{K_{q, t, \rho}\left(z, \bar{Z}_{j}\right) K_{1-q, t, \rho^{-1}}\left(z, \bar{Z}_{j}\right) d z\right\}_{j}$ is complete in $H_{2}^{1}(S)$.

Here we note that for the set

$$
\left\{\frac{\partial^{n} K_{q, t, \rho}\left(z, \bar{z}_{1}\right) K_{1-q, t, \rho-1}\left(z, \bar{z}_{1}\right) d z}{\partial \bar{z}_{1}^{n}}\right\}_{n=0}^{\infty}
$$

for any fixed $z_{1} \in S$, Theorems 4.1 and 5.1 are valid, as we see easily from those theorems. The circumstances are similar for Theorems 2.2 and 3.3 in [6].

6. Szegö kernels with characteristic. Let $U=\left\{U_{\alpha}\right\}$ be a covering of $S$ such that to each $U_{\alpha}$ there is associated a unique local uniformizing parameter $z_{\alpha}: U_{\alpha} \rightarrow C$ and $z_{\alpha}$ and $z_{\beta}$ are analytically related on $U_{\alpha} \cap U_{\beta}$. Then, for a real value of $q$, a differential $f(z)(d z)^{q}$ of order $q$ is defined as a collection of variables which satisfy the transformation laws 
THE EXACT BERGMAN KERNEL AND THE KERNELS OF SZEGÖ TYPE 555

$$
\psi_{\alpha}=\psi_{\beta}\left(\frac{d z_{\beta}}{d z_{\alpha}}\right)^{q} \text { in } \quad U_{\alpha} \cap U_{\beta}
$$

Of course, this function $\left(d z_{\beta} / d z_{\alpha}\right)^{q}$ is not unique, in general and we must show that we can choose them consistently; i.e., such that

$$
\left(\frac{d z_{\beta}}{d z_{\alpha}}\right)^{q}\left(\frac{d z_{\gamma}}{d z_{\beta}}\right)^{q}\left(\frac{d z_{\alpha}}{d z_{\gamma}}\right)^{q} \equiv 1 \quad \text { in } \quad U_{\alpha} \cap U_{\beta} \cap U_{\gamma} .
$$

This is a crucial point, in the treatment of differentials $f(z)(d z)^{q}$ for a noninteger value of $q$ (cf. [8], pp. 249-251 and [2], pp. 215218). In the case of $q=1 / 2$, which is considered as an especially important case, N. S. Hawley and M. Schiffer [2] for the first time had investigated "the Szegö kernel" of half-order differentials and then D. A. Hejhal [3] in the case of planar regions and J. D. Fay [1] in the case of arbitrary compact bordered Riemann surfaces had investigated.

In this final section, we shall see that our results are valid even if in the case of the Szegö kernels with characteristic on an arbitrary compact bordered Riemann surface.

In order to save space, we shall use the same notations and results freely in Chapter VI in [1].

For any even half period $e \in T_{0}$, the Szegö kernel $\sigma_{e}(\bar{x}, y)$ of a compact bordered Riemann surface $\bar{R}$ with characteristic [e] is defined as follows:

$$
\sigma_{e}(\bar{x}, y)=\frac{1}{2 \pi i} \frac{\theta[e](y-\bar{x})}{\theta[e](0) E(y, \bar{x})} .
$$

Here $\theta[e](z)$ is the first order theta function with characteristic [e], $E(y, \bar{x})$ is the prime form and $\bar{x}$ is the symmetric point of $x$ on the double $C$ of $R$ ([1], p. 124, Proposition 6.14 and cf. Chapters I and II). Then $\sigma_{e}(\bar{x}, y)$ is holomorphic in $\bar{x}$ and $y$ except for a pole along $y=x$ and satisfies

$$
\sigma_{e}(\bar{x}, y)=-\sigma_{e}(y, \bar{x})=-\overline{\sigma_{e}(x, \bar{y})} \quad \text { for all } x, y \in C \text {. }
$$

For any section $\Phi$ of $L_{e}$ holomorphic on $\bar{R}, \sigma_{e}(\bar{x}, y)$ has the reproducing property

$$
\Phi(x)=\int_{\partial R} \Phi(y) \overline{\sigma_{e}(\bar{x}, y)} \quad \text { for all } x \in R .
$$

Again $\sigma_{e}(\bar{x}, y)$ is represented by $\sum_{j} \overline{\Phi_{j}(x)} \Phi_{j}(y)$ for a complete orthonormal system $\left\{\Phi_{j}\right\}$ of holomorphic sections of $L_{e}$ on $\bar{R}$. Further we see that $\sigma_{e}(\bar{x}, y)^{2}$ is a single-valued analytic differential on $C \times C$ except for a double pole along $y=x$ and satisfies 


$$
\begin{aligned}
\overline{\sigma_{e}(\bar{x}, y)^{2}} & =-\overline{\left(\frac{\theta[e](y-\bar{x})^{2}}{4 \pi^{2} \theta[e](0)^{2} E(y, \bar{x})^{2}}\right)} \\
& =-\frac{\theta[e](\bar{y}-x)^{2}}{4 \pi^{2} \theta[e](0)^{2} E(\bar{y}, x)^{2}} \\
& =\sigma_{e}(x, y)^{2} \quad \text { along } y \in \partial R .
\end{aligned}
$$

Hence in our case, we obtain the following representations as in (4.1) and (4.2):

$$
4 \pi^{2} \sigma_{e}\left(\bar{z}_{1}, z\right)^{2}=\pi K^{E}\left(z, \bar{z}_{1}\right)+\sum_{\nu=1}^{N} \sum_{\nu=1}^{N} \widetilde{C}_{\nu \mu} \overline{Z_{\nu}\left(z_{1}\right)} Z_{\mu}(z)
$$

and

$$
-4 \pi^{2} \sigma_{e}\left(z_{1}, z\right)^{2}=\pi L^{E}\left(z_{1}, z\right)-\sum_{\nu=1}^{N} \sum_{\nu=1}^{N} \overline{\widetilde{C}_{\nu \mu}} Z_{\nu}\left(z_{1}\right) Z_{\mu}(z)
$$

Therefore for $4 \pi^{2} \sigma_{e}\left(\bar{z}_{1}, z\right)^{2}$, we obtain the same results as

$$
K_{q, t, \rho}\left(z, \bar{z}_{1}\right) K_{1-q, t, \rho^{-1}}\left(z, \bar{z}_{1}\right) d z \overline{d z_{1}},
$$

by a slight modification. In the proof of the main Theorem 3.1, for example we need a nonvanishing section $\Phi_{0}$ of $L_{e}$ holomorphic on $\bar{S}$, but the existence of such a $\Phi_{0}$ is clear. As to this fact, we recall that there exists a single-valued function which is analytic and nonvanishing on $\bar{S}$ except for poles and zeros of prescribed order at prescribed points (cf. [4], § 2.5).

ACKNOWLEDGMENT. The author wishes to thank the referee for his very helpful advice for the expression of this paper.

\section{REFERENCES}

1. J. D. Fay, Theta functions on Riemann surfaces, Lecture Notes in Mathematics, 352.(1973), 138.

2. N. S. Hawley and M. Schiffer, Half-order differentials on Riemann surfaces, Acta Math., 115 (1965), 199-236.

3. D. A. Hejhal, Theta functions, kernel functions and Abelian integrals, Amer. Math. Soc. Memoir, 129 (1972), 112.

4. A. H. Read, A converse of Cauchy's theorem and applications to extremal problems, Acta Math., 160 (1959), 1-22.

5. S. Saitoh, The kernel functions of Szegö type on Riemann surfaces, Kōdai Math. Sem. Rep., 24 (1972), 410-421.

6. - On some completenesses of the Bergman kernel and the Rudin kernel, Pacific J. Math., 56 (1975), 581-596.

7. M. Schiffer and D. C. Spencer, Functionals of Finite Riemann Surfaces, Princeton University Press, 1954, p. 451. 
8. M. Schiffer and N. S. Hawley, Connection and conformal mapping, Acta Math., 107 (1962), 175-274.

Received August 30, 1976.

GUNMA UNIVERSITY

1-5-1 TENJIN-CHO

KIRYU 376, JAPAN 



\section{PACIFIC JOURNAL OF MATHEMATICS}

\section{EDITORS}

RICHARD ARENS (Managing Editor)

University of California

Los Angeles, CA 90024

Charles W. Curtis

University of Oregon

Eugene, OR 97403

C. C. MOORE

University of California

Berkeley, CA 94720

\section{J. DugundJI}

Department of Mathematics

University of Southern California

Los Angeles, CA 90007

R. FinN and J. Milgram

Stanford University

Stanford, CA 94305

ASSOCIATE EDITORS
E. F. BECKENBACH
B. H. NeumanN
F. WOLF
K. YOSHIDA

\section{SUPPORTING INSTITUTIONS}

UNIVERSITY OF BRITISH COLUMBIA CALIFORNIA INSTITUTE OF TECHNOLOGY

UNIVERSITY OF CALIFORNIA

MONTANA STATE UNIVERSITY

UNIVERSITY OF NEVADA, RENO

NEW MEXICO STATE UNIVERSITY

OREGON STATE UNIVERSITY

UNIVERSITY OF OREGON

OSAKA UNIVERSITY

\author{
UNIVERSITY OF SOUTHERN CALIFORNIA \\ STANFORD UNIVERSITY \\ UNIVERSITY OF HAWAII \\ UNIVERSITY OF TOKYO \\ UNIVERSITY OF UTAH \\ WASHINGTON STATE UNIVERSITY \\ UNIVERSITY OF WASHINGTON \\ AMERICAN MATHEMATICAL SOCIETY
}

The Supporting Institutions listed above contribute to the cost of publication of this Journal, but they are not owners or publishers and have no responsibility for its content or policies.

Mathematical papers intended for publication in the Pacific Jaurnal of Mathematics should be in typed form or offset-reproduced, (not dittoed), double spaced with large margins. Please do not use built up fractions in the text of your manuscript. You may however, use them in the displayed equations. Underline Greek letters in red, German in green, and script in blue. The first paragraph or two must be capable of being used separately as a synopsis of the entire paper. Items of the bibliography should not be cited there unless absolutely necessary, in which case they must be identified by author and Journal, rather than by item number. Manuscripts, in triplicate, may be sent to any one of the editors. Please classify according to the scheme of Math. Reviews, Index to Vol. 39. All other communications should be addressed to the managing editor, or Elaine Barth, University of California, Los Angeles, California, 90024.

The Pacific Journal of Mathematics expects the author's institution to pay page charges, and reserves the right to delay publication for nonpayment of charges in case of financial emergency.

100 reprints are provided free for each article, only if page charges have been substantially paid. Additional copies may be obtained at cost in multiples of 50 .

The Pacific Journal of Mathematics is issued monthly as of January 1966. Regular subscription rate: $\$ 7200$ a year (6 Vols., 12 issues). Special rate: $\$ 36.00$ a year to individual members of supporting institutions.

Subscriptions, orders for back numbers, and changes of address should be sent to Pacific Journal of Mathematics, 103 Highland Boulevard, Berkeley, California, 94708.

PUBLISHED BY PACIFIC JOURNAL OF MATHEMATICS, A NON-PROFIT CORPORATION

Printed at Kokusai Bunken Insatsusha (International Academic Printing Co., Ltd.). 8-8, 3-chome, Takadanobaba, Shinjuku-ku, Tokyo 160, Japan.

Copyright (C) 1975 by Pacific Journal of Mathematics Manufactured and first issued in Japan 


\section{Pacific Journal of Mathematics}

\section{Vol. 71, No. $2 \quad$ December, 1977}

Krishnaswami Alladi and Paul Erdős, On an additive arithmetic

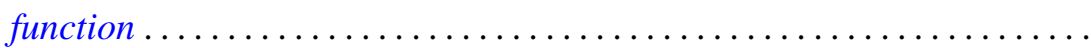

James Bailey and Dale Rolfsen, An unexpected surgery construction of a

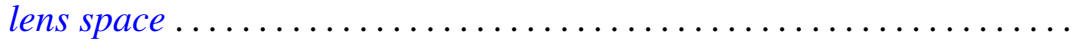

Lawrence James Brenton, On the Riemann-Roch equation for singular

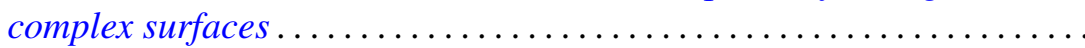

James Glenn Brookshear, Projective ideals in rings of continuous

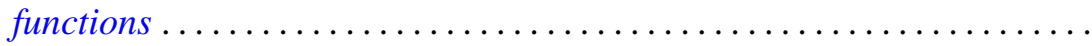

Lawrence Gerald Brown, Stable isomorphism of hereditary subalgebras of

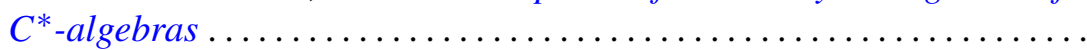

Lawrence Gerald Brown, Philip Palmer Green and Marc Aristide Rieffel, Stable isomorphism and strong Morita equivalence of $C^{*}$-algebras....

N. Burgoyne, Robert L. Griess, Jr. and Richard Lyons, Maximal subgroups

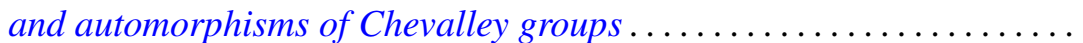
365

Yuen-Kwok Chan, Constructive foundations of potential theory .... 405

Peter Fletcher and William Lindgren, On $w \Delta$-spaces, $w \sigma$-spaces and

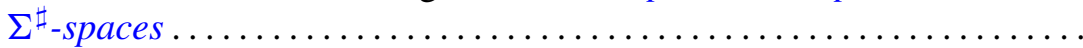

Louis M. Friedler and Dix Hayes Pettey, Inverse limits and mappings of

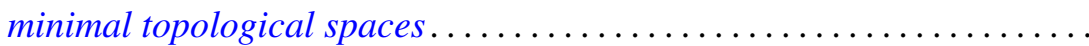

Robert E. Hartwig and Jiang Luh, A note on the group structure of unit regular ring elements.

I. Martin (Irving) Isaacs, Real representations of groups with a single involution ...

Nicolas P. Jewell, The existence of discontinuous module derivations . .

Antonio M. Lopez, The maximal right quotient semigroup of a strong semilattice of semigroups .......................

Dennis McGavran, $T^{n}$-actions on simply connected $(n+2)$-manifolds

Charles Anthony Micchelli and Allan Pinkus, Total positivity and the exact $n$-width of certain sets in $L^{1}$.

Barada K. Ray and Billy E. Rhoades, Fixed point-theorems for mappings with a contractive iterate .......................

Fred Richman and Elbert A. Walker, Ext in pre-Abelian categories. .

Raymond Craig Roan, Weak* generators of $H^{\infty}$ and $l^{1}$..

Saburou Saitoh, The exact Bergman kernel and the kernels of Szegö type...

Kung-Wei Yang, Operators invertible modulo the weakly compact 